\title{
THE ROLE OF THE GANGLION CELLS IN THE SMALL INTESTINE TAKEN IN THE INTESTINAL INTRINSIC REFLEX
}

\author{
Takesi Hukuhara, Tadaaki Sumi And Satoru Kotani* \\ Department of Physiology, Okayama University Medical School, Okayama
}

HukUhara et al.4) showed that the intrinsic reflexes of dog's small intestine consist of two different kinds of reflexes, that is, the mucosal and muscular reflexes. Then they ${ }^{5}$ showed that the mucosal reflex plays an important role in the transport of the intestinal contents. Both reflexes can be abolished by administration of $\mathrm{C}_{6}{ }^{4, \pi}$ ) and the mucosal reflex also by cocainization of the mucosa..$^{5,6}$ ) It can therefore be considered that the mechanism concerned with the intrinsic reflexes may be involved in the intramural ganglion cells. In the present experiment we tried to destroy these ganglion cells. The results are reported in the following.

\section{METHODS}

Dogs are anesthetized with morphine $(3 \mathrm{mg} / \mathrm{kg})$ and urethane $(1 \mathrm{~g} / \mathrm{kg})$. A jejunal loop of length of about $13 \mathrm{~cm}$. is isolated by ligatures from the rest of the intestine, the mesenteric nerves distributing to the loop being severed at their roots. At first the effects of the intrinsic reflexes are confirmed on the loop, the mucosal one being caused by stroking or applying the $0.1 \mathrm{~N} \mathrm{HCl}$ solution to the mucosa and the muscular one by stretching the muscle layers, and then the loop is kept in a state of complete anemia, the procedure being as follows (FIG. 1): The mesenteric artery b and vein a distributing to the loop are isolated carefully at their respective roots,

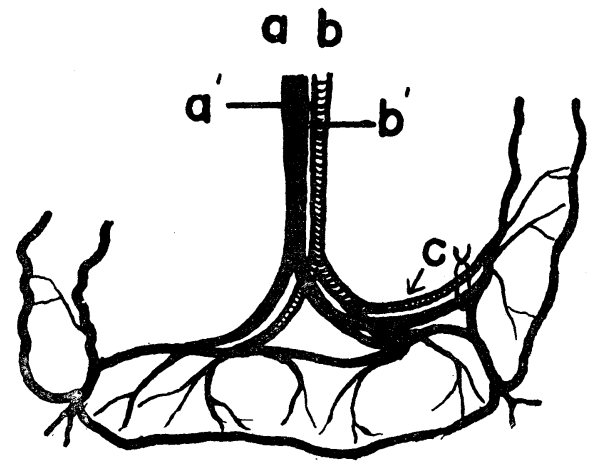

Received for publication November 12, 1960 福原武, 角忠明, 小谷営
FIG. 1. Schema for explaining the procedure how the blood vessels of the intestinal loop is perfused with TYRODE solution. 
and a branch $\mathrm{c}$ of the artery distributing to the part of the intestine adjacent to an end of the loop is also isolated and ligatured at its periphery. After the artery $b$ has been clamped at $b^{\prime}$, TYRODE solution is injected into its branch $c$. The solution perfuses the blood vessels of the loop via the artery and then flows into the vein, thus finally resulting in the expulsion of all the blood contained in the loop. Then the vein $a$ and its branch lying side by side with the arterial branch $c$ are clamped, the loop being thus kept in a state of complete anemia for periods varying from 1/4 to 4 hours or more. With the lapse of time above mentioned the clamps applied to the artery $b$ and vein a are removed to reestablish the normal blood supply of the loop. Thereafter the intrinsic reflexes are pursued at intervals.

A series of chronic experiments are also carried out: After the loop has been kept in a state of complete anemia for a time sufficient to abolish the intrinsic reflexes completely, the animal is allowed to survive for varying periods from 14 to 70 days, the loop being isolated sometimes as THIRY-VELLA loop and sometimes as a by-pass-loop.

At the end of the experiments the intramural ganglion cells of the intestinal loop are examined histologically: The sections are made by cutting the intestinal wall crosswise as well as tangentially to its outer surface, and stained with carbol-fuchsin ${ }^{3)}$ and silver. ${ }^{2)}$

\section{RESULTS}

I. The acute experiments. Where the duration of complete anemia is within the range of 2 to 3 hours, the rhythmic contractions always reappear soon after the reestablishment of the blood supply, being weak in the beginning but gradually becoming vigorous with the lapse of time. On the other hand the intrinsic reflexes are greatly affected by anemia. Even when the anemia is continued for only 15 minutes or so, one of the reflexes, the mucosal one is already completely abolished. This abolishment, however, is transitory and reappears soon after the reestablishment of the blood supply, becoming more and more pronounced with the lapse of time. So long as the period of anemia is limited within about 1.5 hours, the complete recovery of the reflexes is possible, and the longer the period of anemia is, the greater is the time elapsed till the reflexes are recovered completely. When the period of anemia is longer than 1.5 hours, the complete recovery of the reflexes can hardly be expected. One of such examples is shown in Fig. 2. In this case anemia is continued for 1 and $3 / 4$ hours. One hundred and twenty minutes after the reestablishment of the blood circulation the inhibitory response of the mucosal reflex is fully developed, whereas its excitatory response does not recover fully even at 155 minutes.

The mechanism involved in the muscular intrinsic reflex is more resistible against anemia than that involved in the mucosal one. The muscular reflex remains intact, if the duration of anemia is limited within the range of about 1 hour. When the duration of anemia is further prolonged, the reflex is abolished transitorily, but can be recovered fully again after the lapse of time of about 1 and $1 / 4$ 


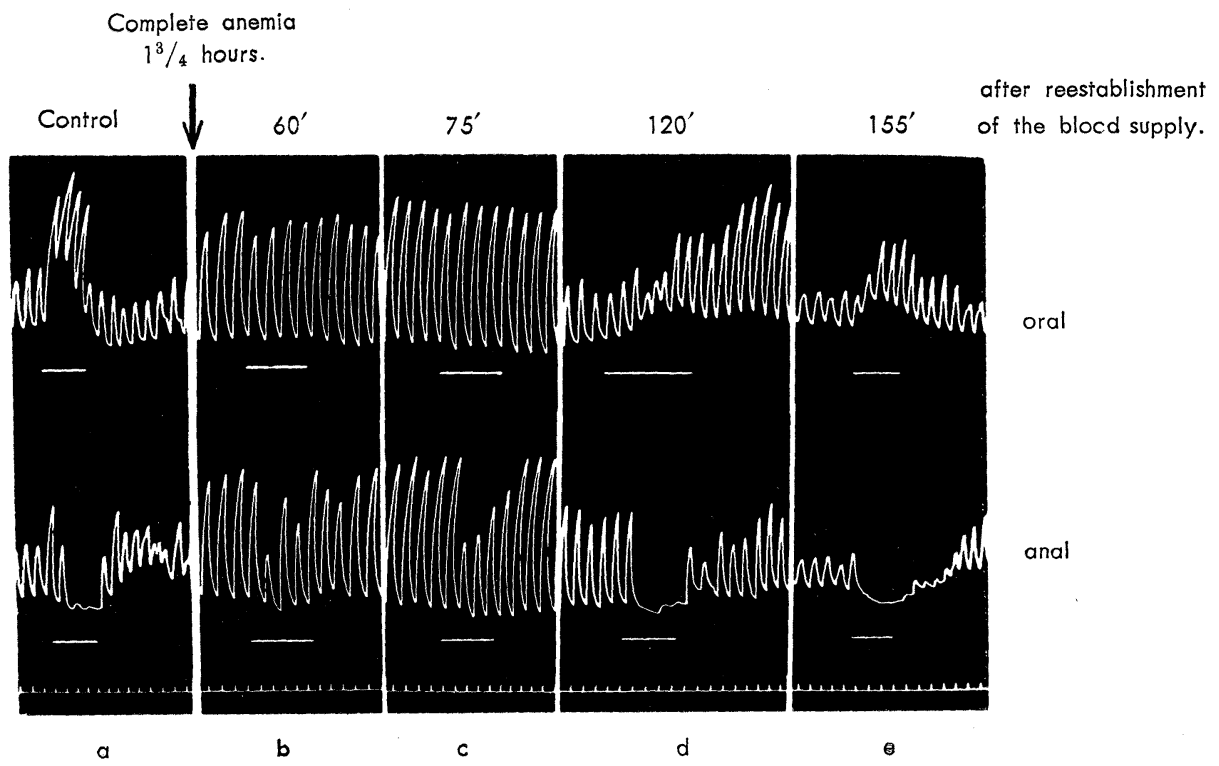

FIG. 2. The course of recovery of the mucosal intrinsic reflex in a denervated jejunal loop whose blood supply has been reestablished after its complete anemia.

a: The mucosal intrinsic reflex responses observed before anemia sets in the loop. $\mathrm{b}$ and $\mathrm{c}$ : Only the inhibitory response is recovered slightly. $\mathrm{d}$ and e: The inhibitory response is recovered almost completely, whereas the excitatory one only moderately.

hours. However, when the anemia is continued longer than about 2 hours, this reflex no longer recovers fully. The Fic. 3 shows the example.

If the duration of complete anemia is plotted against the time elapsed till the reflex responses are recovered fully or incompletely but remained constant, such a diagram as shown in FIG. 4 is obtained. The diagram thus clearly shows the following facts: The longer the period of complete anemia is, the more prolonged is the time elapsed till the reflex responses are recovered, and the muscular reflex is recovered far more rapidly than the mucosal one in which the recovery of the inhibitory response is more rapid than the excitatory one.

When the period of anemia is prolonged to 2.5 hours or more, the intrinsic reflexes, both the mucosal and muscular ones are not at all recovered even in 6 hours after the reestablishment of the blood circulation, while the rhythmic contractions are fairly vigorous.

As regard to the intramural ganglion cells of the loop which is in a condition just mentioned, the histologic examination reveals the following (FIG. 5): The cells are characterized by marked enlargement of the cell body which appears pale due to a remarkable diminution of the chromidial substance. Occasionally there are seen also the vacuoles in the cell body and the nucleus is crowded 


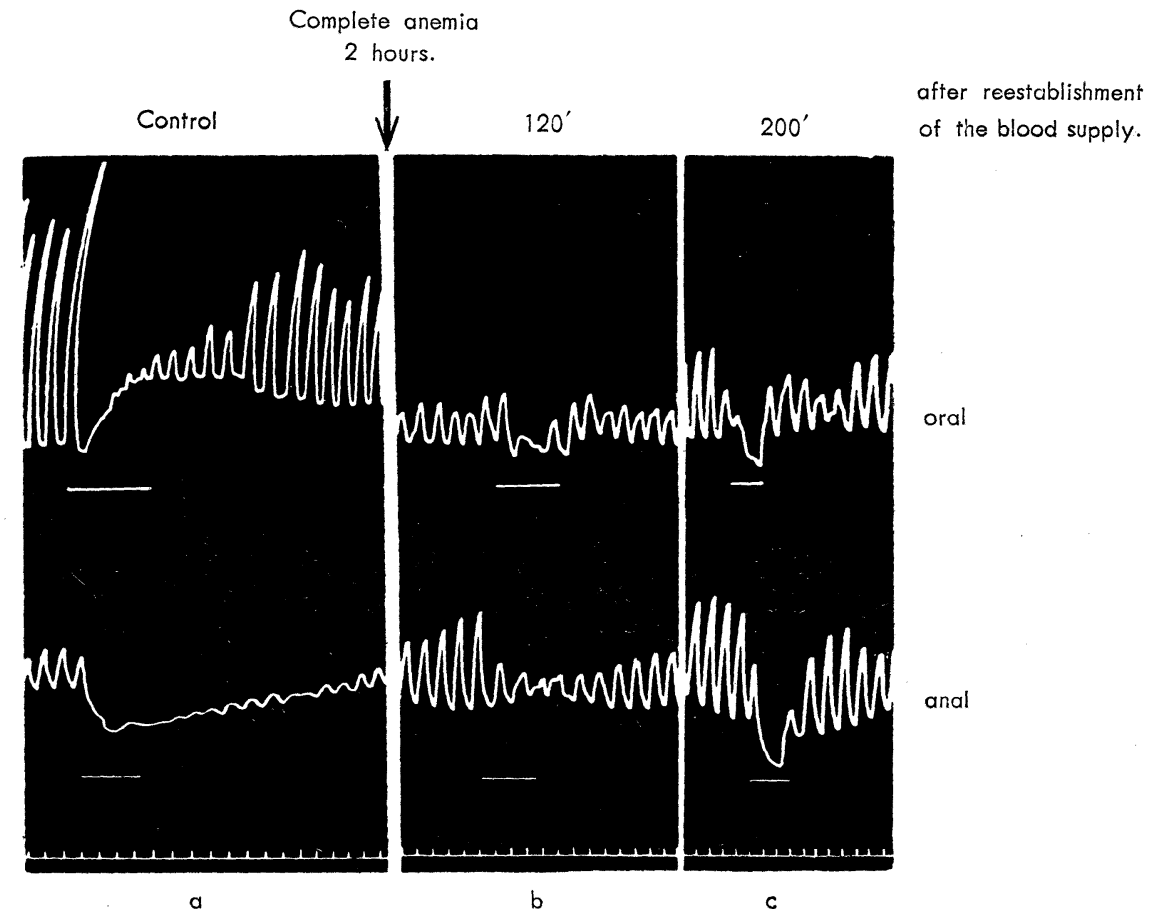

FIG. 3. The course of recovery of the muscular intrinsic reflex in a denervated jejunal loop whose blood supply has been reestablished after its complete anemia.

a: The muscular intrinsic reflex responses observed before anemia sets in the loop. b: The responses reappear moderately. $c$ : The responses are recovered remarkably.

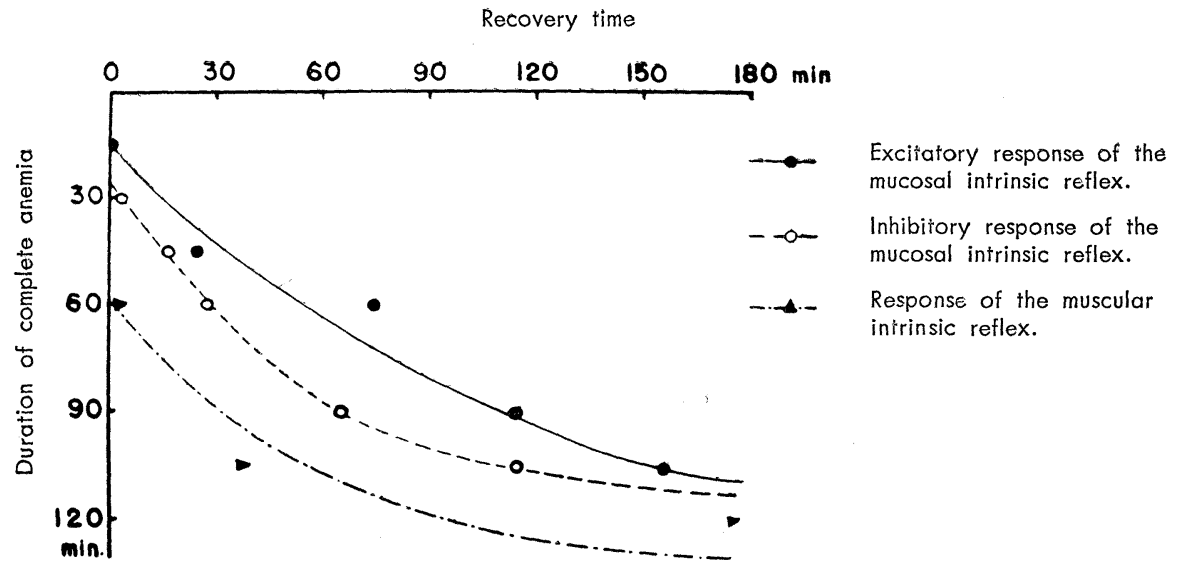

FIG. 4. Relation between the duration of complete anemia and the recovery time of the intestinal intrinsic reflexes. Explanations in text. 


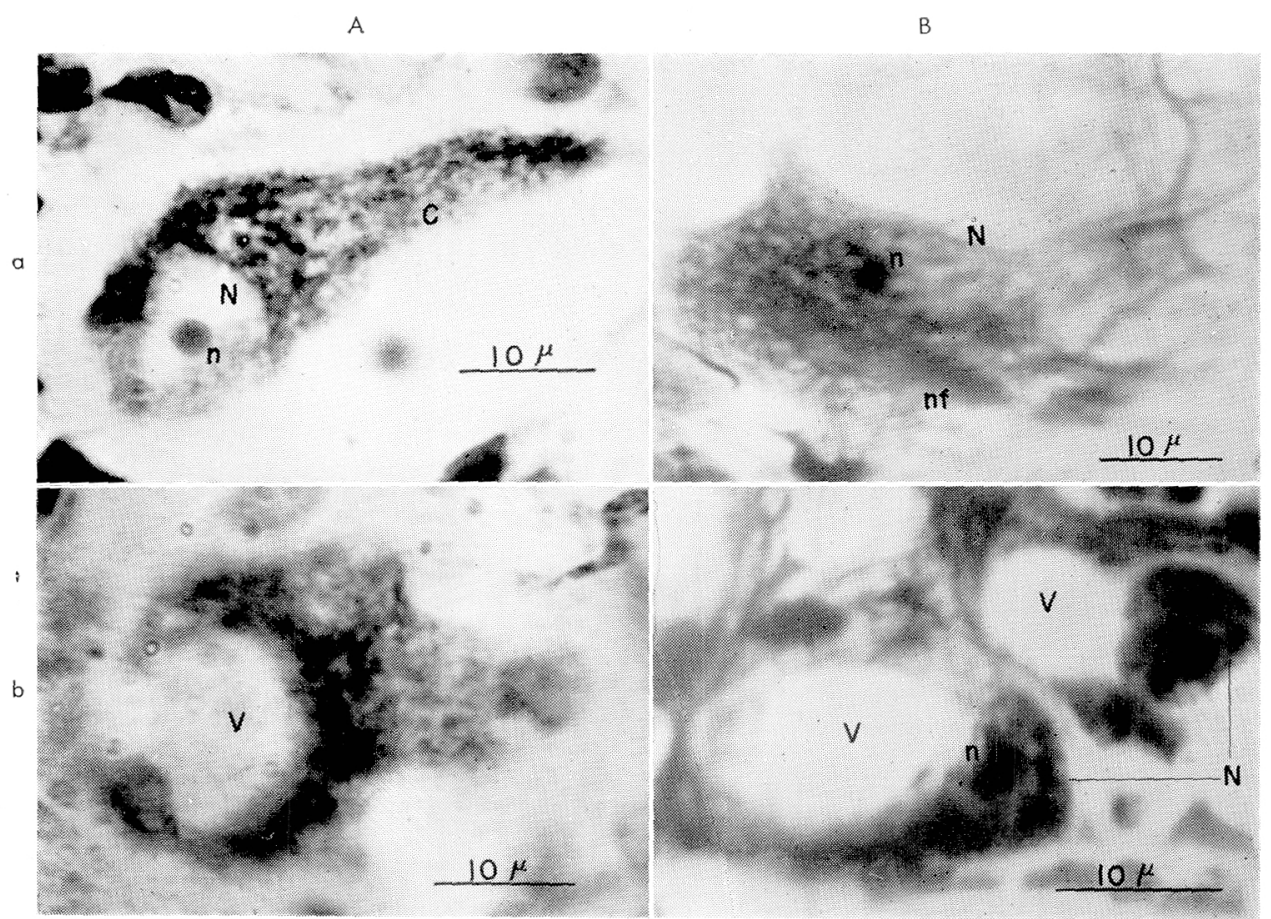

FiG. 5. The ganglion cells in the myenteric plexus of dog jejunum before and after complete anemia continued for 2.5 hours. Tangential section, $20 \mu$.

A : NISSL staining, a: A normal cell (control); the chromidial bodies are distinctly remarked. b: A cell after complete anemia; the cell is homogenously stained and vacuolized, chromidial bodies being diminished remarkably, B: Silver staining. a: A normal cell (control); the neurofibrillar structure and nucleus with a nucleolus are seen. b: cells after complete anemia; the cytoplasm is vacuolized and the nucleus is crowded forward to one pole. structure.

$\mathrm{C}$ chromidial body. $\mathrm{N}$ nucleus. $\mathrm{n}$ nucleolus. $\mathrm{V}$ vacuole. nf neurofibrillar

forward to one pole. In connection with the results obtained in the physiologic experiments, such changes as above are obviously regarded as indicative of physiologic depression of the cells.

II. The chronic experiments. In the acute experiments, as above described, the complete anemia lasting for 2.5 hours abolishes the intrinsic reflexes during succeeding 6 hours despite the reestablishment of the blood circulation. The chronic experiments, however, reveals that the reflexes reappear provided the time of recovery is allowed to elapse over 14 days: In 3 experiments in which 30,63 and 70 days elapsed respectively after being kept in anemic state for 2.5 hours, the reflex response that can be produced, is only the inhibitory one of the mucosal reflex, though it is far less remarkable than that observed when the intestine is intact. The anemia lasting for 3.5 hours proves also to be not 
Where anemia is continued for 4 hours, no sign of the reflexes is recognized even 49 days afterwards. In this case the motility of the loop is worthy of note: In contrast to the rest of the intestine the loop is tonically contracted, and furthermore pulsating rings of tonic constriction are to be seen a few centimeters apart from each other, the waves originating in the rings propagate rhythmically in both directions, interfering with each other and thus making the aspect of the movements of the loop complicated. There can hardly be seen any wave that propagates along the wall of the loop from the one end to the other. Weak stretching of the muscle layers does not produce any recognizable inhibitory response, instead of that a series of rhythmical contractions transitorily occurs, suggesting the enormous rise in the excitability of the muscular tissue. Not only stroking but pressing of the mucosal surface also elicits a series of contractions above and below the spot stimulated. These effects are, however, not regarded as those produced by a reflex mechanism, but as those produced by a mechanical stimulation of the muscular tissue which is so excitable that the least distension stimulus can elicit a series of contractions, since there cannot be observed any change of intestinal motility, when a filter paper moistened with $0.1 \mathrm{~N}$. solution of hydrochloric acid is applied to the mucosa taking care not to press its surface.

Histologically the intramural ganglion cells show in the tangential section the changes parallel to the results obtained in physiologic experiments: Fourteen days after the anemia continued for 2.5 hours the amelioration is not yet re-

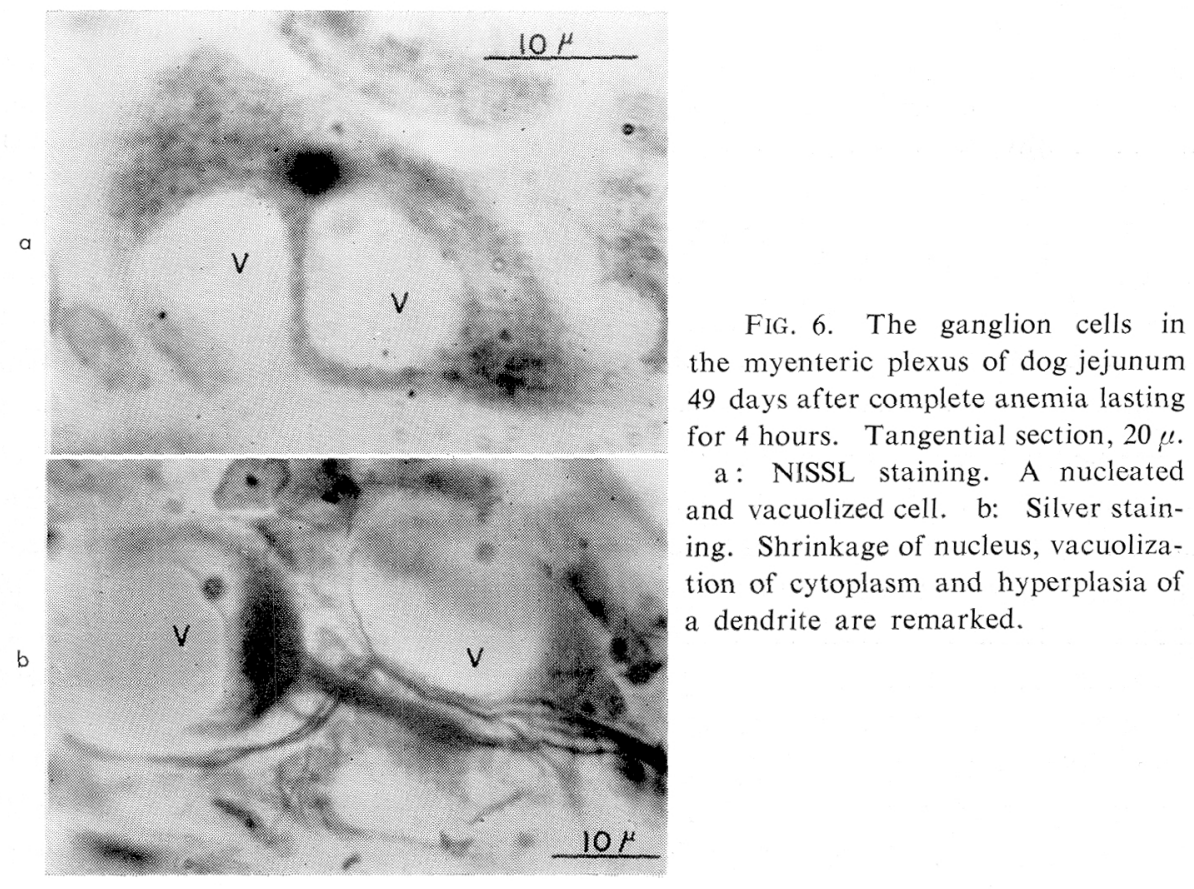


cognized in the aspect of the cells, while 30 to 70 days after anemia there are found a few ganglion cells which nearly show the normal distribution of the chromidial bodies, although both the enlargement of the cell body and irregular hypertrophy of dendrites (in silver staining) are apparently remarked, sparsely among the degenerated cells where diffusely-stained cytoplasm is shrunken and distorted by vacuolization, dangling about the nucleus somewhat like debris of tissues, and frequently the shrinkage and dissolution of the nucleus take place. On the other hand 49 days after anemia lasting for 4 hours almost all the ganglion cells undergo the degenerative changes similar to those described above (FIG. 6).

It is to be noted further that the experiments, in which anemia is continued for 5 hours, are not successful; in one case the animal died of the perforation of the loop 3 days after the operation, while in the other it died of general debility 2 days after the operation.

\section{DISCUSSION}

From the results described above it may be considered that complete anemia lasting for 4 hours destroy all the intramural ganglion cells to such a degree as to incapacitate them from ever recovering again, resulting in a complete abolishment of the intrinsic reflexes. From these facts it may be reasonable to assume that the elicitation of the intestinal intrinsic reflexes is attributable to the mechanism involved in the intramural ganglion cells. It can be surely perceived that in such intestine as mentioned above rhythmical contractions occur here and there, although the polarity is lost in these contraction waves. From these facts it can be unequivocally concluded that the muscle of the intestine has an ability to contract with its inherent rhythm independently from the ganglion cell.

CANNON and BURKET ${ }^{1)}$ produced anemia by compression of a part of the intestine and found that when compression anemia lasts for 3 and $1 / 2$ hours or longer, 16 days afterwards all nerve cells disappear in the compressed parts. Their histologic observation was performed on the cross-section of the intestine. It is worthy to note that the cross-section is unfavorable for observing the aspect of degenerated cells such as above described, because it cannot reveal the whole aspect of the cell, but only its limited parts which appear apparently somewhat like debris of tissues so that one might fail to identify it as parts of a ganglion cell. And this might be the case when CANnon and Burket said that all the ganglion cells disappeared 16 days after the compression anemia. Their results on the histologic examination thus on the whole agree with those obtained by us. However, in the point of their contention that disappearance of nerve cells almost invariably results in loss of motility, we cannot very well agree with them, because in the present experiments, on the contrary, it has been proved that the contractions surely appear independently from the ganglion cells. In addition, 
it should be noted that they actually, in one case, observed contractions occurring in the stomach and proximal colon after anemia that lasted for 4 hours.

\section{SUMMARY}

1) A denervated jejunal loop of dogs is kept in a state of complete anemia by perfusing the blood vessels of the loop with TYRODE solution for varying periods from $1 / 4$ to 4 hours. The blood circulation of the loop is then reestablished, and the intestinal intrinsic reflexes are traced at intervals. At the end of the experiments intramural ganglion cells of the loop are examined histologically.

2) Where complete anemia of the loop is continued for 4 hours, the intrinsic reflexes remain abolished permanently, almost all the intramural ganglion cells of the loop undergoing the degenerative changes. It may be thus confirmed that the intrinsic reflexes are produced by a mechanism involved in the intramural ganglion cells.

3) There obviously occur the rhythmic contractions in the loop described above. Consequently it may be concluded that the rhythmic contractions of the small intestine are essentially of myogenic origin.

\section{REFERENCES}

1) Cannon, W. B. And Burket, I. R. The endurance of anemia by nerve cells in the myenteric plexus. Amer. J. Physiol. $32: 347-357,1913$.

2) Feyrter, F. Über die Pathologie der vegetativen nervösen Peripherie und ihrer ganglionären Regulationsstätten. Wilhelm Maudrich, Wien. S. 173-180. 1951.

3) GưR, E. A practical manual of medical and biological staining techniques. Leonard Hill, London. p. 79-80, 1956.

4) Hukuhara, T., Yamagami, M. and Nakayama, S. On the intestinal intrinsic reflexes. Jap. J. Physiol. 8: 9-20, 1958.

5) Hukuhara, T., Nakayama, S. And Sumi, T. The role of the intrinsic mucosal reflex in the fluid transport through the denervated intestinal loop. Jap. J. Physiol. 9: 406$418,1959$.

6) Hukuhara, T., Nakayama, S. And Nanba, R. The effects of 5-hydroxytryptamine upon the intestinal motility, especially with respect to the intestinal mucosal intrinsic reflex. Jap. J. Phsiol. $10: 420-426,1960$.

7) Hukuhara, T., Nakayama, S. And Nanba, R. Locality of receptors concerned with the intestino-intestinal extrinsic and intestinal muscular intrinsic reflexes. Jap. J. Physiol. 10 : 414-419, 1960. 